\title{
Study on the Expression Forms and Techniques of Color in Oil Painting Creation
}

\author{
Lizhi Yang \\ Hetao University, Bayannaoer Inner Mongolia, 015000, China
}

Keywords: Oil painting creation, Color, Form of expression, Skill, Study.

\begin{abstract}
Color is an important language and expression method in creation of oil painting; it have strong artistic expression power, can fully reflect the independent aesthetic value of oil painting. meanwhile, the proper use of color will directly effect the value of the painting, and exploit expression power of the color in oil painting creation, to ensure more personality and the times spirit of the painting
\end{abstract}

\section{Introduction}

In the creation process of oil painting, color plays an important role, it is an important artist method to express personal feelings. Under the development of modern optimization of creative approach, the color has gradually highlighted its importance.. Color can strengthen the artistic effect of the work, by making a deep visual impression, to ensure that the oil painting pieces can make better charm and appeal reflection.

\section{The overview of color in Oil painting}

\section{The color of oil painting in the classical period.}

In the western countries, especially during the period of the middle ages, the position of oil painting is almost identical with sketch, and the expression of color is constantly weakened. Hegel call his own painting style as "sketch "painting, painting and drawing basically alike. Thus, at the time, the form of oil painting color performance is almost no attention.

\section{Color presentation technology in impressionism.}

In nineteenth Century, the impressionism appeared in France. That become a key point for the history of color in oil painting. Impressionist paintings featured by bright expression of light and color,then make the color standing out of shade and form, as so to full fill the independence of color. Oil painting to achieve the perfect transformation from the sketch to the color, but also in the color contrast, the intention to use different techniques, and then to better the idea of light and color in the painting. And the painter does not just stay in the objective description in the nature, but gradually began to focus on personal feelings and practical experience, so that the subjective color in the oil painting established the status of. Therefore, in this period of the creation of the painting, the artist's own ideas and aesthetic is perfect into the creation of oil painting.

\section{The status of color in the creation of contemporary oil painting.}

The contemporary oil painting has diversified concrete manifestation and always in a state of constant innovation. Among western contemporary oil painting, expressionism have gradually emerged to be the mainstream. Personal spirit and the consciousness is fully exhibited. Meanwhile the performance of the color become more free, thus showing a westernization and personalized features. And in the process of development of oil painting, artists began to pay attention to the personalized characteristic color, and through on color change of direct experience and observation, to enable personal work more with spiritual influence and art expressive strength.

\section{The combination of Chinese and Western painting.}

Certain changes have been made after connection was been made between China and the West; it is switching gradually from realism to performed as well as from decorative painting into an abstract painting.during absolve the west techniques, Chinese oil painting also gain great development. In oil 
painting, Chinese oil painting conduce full use color tech ques, and filly integrated love for life fully into work, and express the inner world of the anther and enhance the painting with more vigor and vitality.

\section{The significance of color art in oil painting creation}

\section{Color can renew the life.}

In creation stage of oil painting creation,is the reflection of real the life in oil painting ; the reflection is more than simple mirroring but a reproduction of the process in the original basis. In the creation of oil painting, color and emotion has a strong connection, it is a kind of inspiration from life and emotion to fuse and collide. Therefore, even if do not understand the art of appreciation, but also from the color of pure experience to the vitality and beauty of life.

\section{Color can reflect the level of space.}

In oil painting,different spaces can be shown through the color. In order to highlight the distance far away and the middle distance of the air is relatively thick,all the color have to be green gray in some level under the sun.. At the same time, we can also place one subject in front of the other, so as to display the distance by the overlap between objects. Show near things need to draw in a lower position, the shape is relatively complete, far away from the show is just the opposite of things. In addition, can also be through the mediation of color changes in temperature as well as the size of the shape to highlight the different levels of the screen, so as to highlight the three-dimensional sense of the picture.

\section{Color bring activities into in the picture.}

The theme of and between painting and color between the two are interlinked, can embody the effective of nonstaining by shapes, color and bring activism into the painting. As everyone knows, things can be expressed through the color and shape of the picture, For example, in the process of sketching, black is the main color of the shadow; and in the creation of oil painting, the dark block is the main tone of the cold and warm tendency. The former, clear colors, black and white; the latter, color showing a variety of features, including green ash, red ash and warm gray and cold ash, the active picture to an indispensable role. Not only that, in the process of application of the shades of color, the processing of oil painting, enough to achieve the desired effect. Among them, the dark is the main part, in the background of bright colored foil to picture of the expression to be further filling and rich.

\section{The expression forms and techniques of color in oil painting}

\section{Color expression.}

a). Space Ability of color space.In the process of the creation of oil painting, space creation and expression can be used to make changes in the perspective of form to express, but also can use the color changes to better performance of the screen space. First of all, the color distance method, this is a kind of perspective. If the landscape is getting farther and farther, the middle air layer will become more and more thick. As a result, the air will show a slight bluish green in the sun, making the object more distant color lower, but also gradually become weak contrast. To show the relationship between the above colors in the picture which is called the color distance method. And the color perspective is the first in the "Nationalism" in the work of the show, the background of the work is the use of green gray. Secondly, the plane overlapping method is also used in color space, and at the beginning is the perspective of law. Is the scenery in which a number of planes, one in front of the other, and the use of the object before and after the overlap of the relationship between the distance to represent a painting. The closer the distance, the more complete the form, and the position is relatively low. However, the distance is just the opposite. In Gauguin landscape paintings "dry haystack" which use this method to be shown, through the use of depth and color changes in temperature, or is the size and shape of a variety of color to build a level before and after picture, and reflect the color expression of space effect. 
b). Color shaping body make it more divide.In painting, color is an important expression of language. The color as the art form of painting language, we must ensure that they meet the "form" and "theme", only in this way can we truly to write to God and really play the important role of color in the design. First, oil painting, color is important is to warm and cold colors of body shape is like drawing shadows need to use black, and in the middle of the painting color, we need to use with the tendency of cold and warm dark blocks. In the sketch, a discrepancy is gray black and white lightness, and in color, the gray cold and warm gray, red and gray, gray green and silver and so on. Also in the sketch, high light fingered is paper white, however, in color, high light the vocabulary represents is the sky color or other light source color reflection. In the "girl and peach" works, character's face and hands, and pink dress belong to warm color block, thus work environment and cold gray formed obvious contrast. In addition, the dark figure and peach are warm, and the light is cold. On this basis, in paintings, deep color of the back of a chair, brooches and hair. These dark blocks a interludes, thus making the picture contrast of changes in temperature is more intense, and the picture is more active. In addition, in the blue eyed girl "this works also goes through between red and green to produce visual effects. At the same time, the use of color and size of the thrust, and achieves the ideal effect of a rich picture and unified. Secondly, an effective processing method can make full use of the color contrast depth as the picture effect. The main thing if you can use a dark, light background. If the subject matter is bright, it should match the dark background. If the contrast is relatively strong, then the screen will gradually increase the degree of intuition. In the queen Marit, black characters mast dress, and deep color of hair, and the formation of the pyramid shape. And people face and white decorative collar, including the body before hand, all use white color, as a result, between clothes and produced strong contrast. On this basis, the picture of the brown background also uses the gray level of color to better bring out the main body.

\section{Color can express motion.}

a). Artistic conception of color In the process of oil painting, the use of color is similar to the notes, while the color is the movement, the color changes with different characteristics, but also in harmony and harmony to achieve unity. In the oil painting creation, medium tone can not only comprehensively shows the color changes of the rich, but also through the use of color to express their feelings, the use of color to convey the expression. So, artists have to good for color detection and effective application, ensure the mood of the people to form the resonance, makes the viewer can also deeply infected and produce unlimited life, eventually into the aesthetic process. Among them, the autumn is the harvest season, so, yellow is the color of the harvest. Therefore, in the creation of oil painting, in general, the use of yellow to symbolize prosperity and harvest. The green tone is expressed by life and hope. In addition, blue and purple and other cold colors, you can represent the cold and cold. In "blood in May, 2008" in this work, the artist Yang Feign and Lee on the full use of color to enhance the expression of the main effect. In images of the works, the author uses the blue and purple frisson, will be after the big earthquake pain and sadness in the hearts of people perfect foil, through the screen can feel when people's minds, and seem to be able to hear the loud cry, that whine and cry for help, lost loved ones that sad and helpless. And in the picture also used the red and green, the rescue team red uniforms, and the people's Liberation Army green uniform. It also gives people hope. The whole works to show the Chinese people in the face of disaster that spirit of unity is strength, and also reflects the tragic scene of people struggling to disaster. In addition, the artist is like the warm colors to reflect the sun's bright feeling, while Van Gog likes to use the yellow to express the kind of people who desire for life. In addition, are more adept at using Gauguin rich and full complement tone to show that primitive and mysterious.

b). The artist likes to use color to show a person's one kind of thought and the yearning for the future, and thus better express their own ideals and a variety of emotions. In the work of "sunflower", the need to give the color of the soul. This work is not a natural portrayal of the reality, but the artist's own life for the spirit of the show. Van Gogh, by using the high purity of the yellow, effectively showing the kind of desire for life and the pursuit of life. In the picture, the color contrast is very strong, and very simple. And its color is also the same as its own display of the hot sun. In the red in the harmonious "works, screen color most of them use a solid and the refined color combination, but 
also makes the picture color effect is more intense. In the middle of the work, indoor red forms a whole tone, at the same time, the window blue and green and red form a contrast. In addition, the orange and yellow sash, the comparison becomes relatively moderate. The window of the house use is pink, in this way, with the red better echo.

\section{Conculsion}

To sum up, in the world of oil painting, the use of color is very important. Through the use of color to better express the form and create artistic conception. At the same time, color can create a better atmosphere, and express emotion, so that the audience can better integrate into the aesthetic realm. Thus, in the creation of oil painting, color is an important means and soul.

\section{References}

[1] $\mathrm{Cu}$ Junmei.A brief discussion on the role of color in oil painting creation and the study, Art education ,2011(9):36-36.

[2] Wu Zhigang.Oil painting in the teaching of oil painting in the color of the performance of the art, Art, 2012(2):95-95.

[3] Gaohui .The study of the color representation skills in oil painting, Journal of Hubei College of science and technology ,2013,33(12):141-142.

[4] Guo Guoren.Analysis on the role of color in oil painting and the expression of emotion, Art education research ,2015(23):47.

[5] Bu Zhongfeng, Jiang Dongmei The emotion and color, Art education in oil painting creation .,2014(24):31-31. 